\title{
Research on Database Programming Technology Based on Computer Software Engineering
}

\author{
Ping $\mathrm{Xu}^{1, \mathrm{a}}$ \\ ${ }^{1}$ School of Information Science and Technology, Taishan University, Tai'an, 271000, China \\ ${ }^{\mathrm{a}}$ email
}

Keywords: Database, Programming technology, Computer software engineering

\begin{abstract}
With the development of computer, the function of database is more and more important. According to the needs of computer performance and practical application, the establishment and access of database are different. This paper discusses the methods of direct establishment and indirect establishment of database and analyzes the key technologies of database access to provide some references for the relative researchers.
\end{abstract}

\section{Introduction}

Software engineering involves programming language, database, software development tools, system platform, standard, design mode of development can effectively improve the quality of products and reduce the maintenance cost according to the characteristics of the target, also greatly improve the development efficiency. In the current software engineering development, the database application system development proportion is bigger, is also more common. The basic features of database application system design are object oriented, operation visualization and graphical interface. For software engineering in the management of database programming, established in the early to have clear direction, completed the basic framework design need to take measures to improve the function, to supplement the function of software and improve their defense capabilities, so even in the network environment, to avoid being the virus attacks, to ensure data security, and the update rate of the information in the database can be achieved using standard requirements. The establishment of the database is based on the programming technology to carry out, for some technical problems, through the coordination between functions, can better avoid technical problems, at the same time in software engineering is put into use to maximize the use of database resources, also can realize the automatic update software in network environment detection. The database software development of traditional software engineering process is often based on single aspects of research, ideas or focus on software engineering, or to face relatively like this, or simply consider the database technology, there is less research on the development of comprehensive database construction. At present, software development is combining engineering ideas with technology oriented and database design techniques.

\section{Establishment of Database}

Direct Establishment. A database is a collection of tables. A relatively fixed table set for managing relationships. It can contain one or more tables, relationships, views, and stored procedures. A table file that has one of the following two states: a database table associated with the database, and a free table that is not associated with the database. Most of the two operations are the same and can be converted to each other. When building a database at first, you must first understand the structure of the database. A database file consists of two parts, one part is the data structure in the establishment of user defined library of each field name, field type and field width etc. is called the library file, stored in the front or library file for the library header. The second part is called the recording part, which stores the recorded data. The field values of numeric fields are decimal data, and they are divided into two types: integer and decimal. The date field value is the date data, the system fixed its 
width is 8 bytes, the user has no right to change, the system default format is American format. The date data type can carry out the following operations: a date plus or minus a decimal integer to a new date, two date subtraction a decimal integer. In the operation process, the system automatically guarantees the carry relation of months, days and years. The logical field width is fixed to 1 bytes, and the user has no right to change it. To store logical values, you can only take two values: logical truth or logical false. When a request to build a database, the structure of the database file can be established with this command after analyzing the structure of the library file.

Indirect Establishment. The indirect creation of a database is not a direct description of each field from the keyboard, but is otherwise obtained from an existing library file or library file structure description file. The database structure description file is defined by the field name, type, width, and decimal four parameters, regardless of the content of the library file, but the description file will not change. Therefore, you can save the structure description of a database, that is, the structure description file. The file name is the structure description file name, it is actually a special library file, but the field is fixed, increase its record is equal to the increase of other library file field structure description, can create a new database using this file. We can build a database file structure with an existing library structure description file. Using this command, you can build database files without entering the field name directly. This method is commonly used in general database programming. For a new database to be built, if the library structure is the same or part of another existing database structure, a new database can be built with the existing database. The record number is used to identify the physical order in which the data is recorded in the table file. A record pointer is an indicator that always points to the record in the current table that is being processed by operation. This record is called the current record. If you want to process a record, you must move the record pointer so that it points to the record. At any point, a pointer can point to only one record. Use this command to generate a specified new database structure in the currently open database file, specified by the specified field table name. If the field name is omitted, the new library structure is the same as the currently open library structure.

\section{Access to Database Files}

Selection of Database Storage Models. With the advent of computer storage devices that enabled computers to store data, data management software was born in this context. But restricted by the technical condition at that time, the data can only be stored in the external storage device in the file as a unit. People have developed an operating system with an interface to manage the stored data. Database storage mode selection needs to be done after the partition, and the storage function may have different levels of functional problems. This database storage mode choice is the definition of access to user permissions in the software, the use of the process, to ensure the security of internal information, will define the user's access rights, there are also differences that different levels of users can login to the database interface, information security have been. On the basis of the file access selection based on file type, and more efficient, to achieve the above functions in the program period to focus on design, according to the type of information stored in the database to make a choice, to avoid a deeper problem, and help the optimization of resources, to achieve more ideal effect in the process of. When different resources are in use, they need to transfer the internal information of the database according to the instructions received to optimize the use of resources. With the popularization and application of computers, more and more data can be stored by computers, and people have higher requirements for database storage technology. Enterprise users want database storage technology to have a high level of sharing capability. Data storage technology in this period has been greatly developed. Now the database storage technology, to a great extent, is also in accordance with the standards of this period to develop accordingly. With the development of database itself, a lot of new database storage technologies have appeared in the world.

Protection of Database Files Encryption. Data is the core asset in the information system, and the loss, damage or leakage of data may bring immeasurable losses to the enterprise. Encrypting sensitive data is one of the core methods in data security protection. There are two types of data types, 
one is unstructured data, such as documents and pictures, and the other is structured data, such as data in a database. The data of these two forms are very important, and encryption protection is needed. Structured data, however, usually contains very centralized and valuable information, so it is important to encrypt and protect it. Moreover, the database management system used by most of the domestic foreign products for safety, controllable consideration, completely replace the present stage of foreign products. Facing the severe reality of frequent leakage of sensitive data, although encryption and decryption process will damage the efficiency of database usage, it is still necessary to protect the database. Database encryption can significantly improve database security. After encryption, the data is stored in ciphertext, which prevents the data from being exposed directly, and enhances access control of encrypted data, which greatly reduces the risk of data leakage and malicious destruction. In the operating system, the file drive layer encrypts the storage files of the database and stores them on disk. When the database accesses the storage file, decrypt it again. This method is also transparent to the database itself, and the database management system does not feel the existence of encryption and decryption. This encryption prevents loss of disk and sensitive data leakage caused by copying files.

Comparison of Data Storage Methods. Database storage technology for paper, traditional storage technology, has a revolutionary effect, because the paper storage data, is vulnerable to water and fire disaster, and data loss Cardiff, human civilization from text, he recorded a large number of historical information, but with the passage of time, a lot of the data are damaged, causing serious losses to human civilization, and database storage technology can well avoid this problem in the database environment, information will be converted into electronic form, stored in the computer hard disk, hard disk for storage, than paper books and other simple more, the environment is relatively low, some of the new server memory, even with fireproof performance, and the data in the database, can use computer is very simple The copy, at present a lot of enterprise database, in order to ensure maximum safety of data, will establish an image database, regular backups of the information in the database, if the database problems, you can restore the way to restore the original data. Storage performance is stable, from using contrast method in the process of study, observe the operation status of the software function if there is unstable phenomenon, and from a technical point of view to discuss preventive measures. The system for rapid screening of the received information in the process of operation, will be one of the useful information is classified, so can be used according to the needs of the rapid mobilization of information in the database, the software is put into use can also be updated to function according to the operation requirements, implementation of this method also need to cooperate with each other between the various systems.

Selection of Database Programming Technologies. For database programs, the most important function is to store data. Usually, a database program is divided into several modules, in which the core module is the database storage technology, while the other modules perform the functions of inputting, modifying and invoking data in the database. Although these functions are indispensable, but as the core of database program, database storage technology should be paid enough attention to by programmers in the programming phase. In the actual database programming, most writers will pay attention to this problem, in the preparation of data entry, modify and call modules, data storage technology will be at the core. If you can improve the efficiency of data storage, you can modify other modules. In the selection of programming technology, we should discuss the stability of software function, observe the optimization of resource utilization in the system design, and the possible instability of function. According to the systematic function of the more common problems, in the programming stage technology selection can using contrast method, observation of the stability of the system function, data transmission found inaccurate to resolve to take timely control measures, prevent the function of the software of large area paralysis, affect the normal use of the work. Inspection work carried out is to solve these questions of technology choice for the detection of the stability design of a software, is a safe and suitable environment for the operation of the system to create, in such an environment can solve the problem of stability operation, and meet the system needs of the working environment. There is a great deal of relationship between software function stability and the choice of programming technology. Therefore, when choosing programming 
methods, we should consider whether we can solve the problem of optimizing the utilization of this technology. If you do not follow this idea for database programming, then write to the data entry staff may be convenient, and modify the database storage type, while improving the efficiency of inputting data, but the data security is not guaranteed. For large and complex databases, data storage efficiency and management are very important, and data entry, modification and call are not the core content of the database. Therefore, only the database storage technology as the core of database programming and programming under this idea can make the database program work best.

\section{Conclusion}

With the development of software industry, programming has become a hot industry. The emergence of various high-level languages greatly facilitates the design and compilation of programs. This paper analyzes the establishment method of database and the key technology of database file access and provides reference for programmer of database based on software engineering.

\section{References}

[1] David O, Ascough J C, Lloyd W, et al. A software engineering perspective on environmental modeling framework design: The Object Modeling System[J]. Environmental Modelling \& Software, 2013(39): 201-213.

[2] Xia Xuegang. Based on software engineering database application system development process norms [J]. Science \& Technology Information, 2009(1): 481-482.

[3] Zhang Tianyi. System design and development based on Software Engineering [J]. Electronic Test, 2015(20): 23-25.

[4] An Hongmei, Reformation and Practice of Database and Java Database Programing course [J]. Science \& Technology Vision, 2012(21): 8-9.

[5] Li Nan, Feng Zhaoyu. Application of Database Technology in Improving the Engineering Design Quality [J]. Contemporary Chemical Industry, 2017(1): 149-152. 